\title{
COOL SUPERGIANTS IN THE SOLAR NEIGHBORHOOD
}

\author{
M. JURA \\ Department of Astronomy \\ University of California \\ Los Angeles CA 90024 USA
}

\begin{abstract}
We have identified 21 mass-losing red supergiants (20 M-type, 1 G-type, $\mathrm{I}$ $>10^{5} \mathrm{~L}_{\odot}$ ) within $2.5 \mathrm{kpc}$ of the Sun. These supergiants are highly evolved descendants of main sequence stars with initial masses larger than about $20 \mathrm{M}_{\odot}$. The surface density projected onto the plane of the Milky Way is between about 1 and $2 \mathrm{kpc}^{-2}$. Although with considerable uncertainty, we estimate that the mass return by the $M$ supergiants is somewhere between 1 and $31^{-5} \mathrm{M}_{\odot} \mathrm{kpc}^{-2} \mathrm{yr}^{-1}$. In the hemisphere facing the galactic center there is much less mass loss from $M$ supergiants than from W- $R$ stars, but in the anticenter direction, the $M$ supergiants return more mass than do the $W-R$ stars. The duration of the $M$ supergiant phase appears to be between 2 and $410^{5}$ years. During this phase a star of initially at least $20 \mathrm{M}_{\odot}$ returns perhaps 3 to $10 \mathrm{M}_{\odot}$ into the interstellar medium.
\end{abstract}

\section{INTRODUCTION}

For single stars in the mass range between 25 and $60 \mathrm{M}_{\odot}$, Chiosi and Maeder (1986) propose the following evolutionary scheme: 0 star $\rightarrow$ Blue Supergiant $\rightarrow$ Yellow/Red Supergiant $\rightarrow$ $\mathrm{W}-\mathrm{R}(\mathrm{N}) \rightarrow \mathrm{W}-\mathrm{R}(\mathrm{C}) \rightarrow$ Supernova. If the mass loss rate is sufficiently low, the W-R phase never occurs, and the star becomes a supernova directly after the red supergiant phase. This scheme ignores mass transfer in binaries which can also lead to the formation of a W-R star (Maeder 1981, Massey 1981, Schulte-Ludbeck 1989). In any case, by comparing the relative numbers of red supergiants and W-R stars, and estimating the mass loss rates from the red supergiants, we can constrain this theoretical picture.

One advantage in studying luminous stars is that they are conspicuous. We are interested in sampling a sufficiently large volume to average over local fluctuations in the surface density of stars yet with sufficient sensitivity to be complete. By analogy with studies of W-R stars (Abbott and Conti 1987), we consider red supergiants within $2.5 \mathrm{kpc}$ of the Swn (Humphreys and McElroy 1984). This is the same volume for which there appear to be reasonably complete catalogs of W-R stars (Conti et al. 1983, van der Hucht et al. 1988)

Cool stars emit most of their energy at wavelengths longward of $1 \mu \mathrm{m}$. In addition, there is less interstellar extinction at infrared than at optical wavelengths, and therefore, infrared 
sky surveys are the most comprehensive ways to identify candidate red supergiant stars in the solar neighborhood although optical spectroscopy is still normally required to establish the nature of the star.

One major observational difficulty is to separate the red supergiants that are descended from massive stars from the very luminous red Asymptotic Giant Branch, [AGB], stars (Iben and Renzini 1983) that are descended from intermediate mass main sequence stars. One approach is to restrict the analysis to stars with luminosities in excess of $10^{5} \mathrm{~L}_{\odot}$, well in excess of the maximum luminosity on the AGB of $610^{4} \mathrm{~L}_{\odot}$. Stars with $\mathrm{L}>10^{5} \mathrm{~L}_{\odot}$ have main sequence progenitors with masses greater than $20 \mathrm{M}_{\odot}$.

During the past decade, considerable progress has been made in determining the mass loss rates from red stars by using molecular and infrared data (see, for example, Jura 1989, Olofsson 1989). All the identified high luminosity red supergiants - even the G star $\rho$ Cas (Jura and Kleinmann 1990a) - exhibit circumstellar dust and are thought to be losing mass.

\section{THE CATALOG}

Catalogs of candidate red supergiant stars in the solar neighborhood have been given by Humphreys (1978), Elias, Frogel and Humphreys (1985) and White and Wing (1978). Most of these stars lie in the zone covered by the Two Micron Sky Survey, TMSS, (Neugebauer and Leighton 1969) which was complete in the declination range of $-33^{\circ}<\delta<81^{\circ}$ to $\mathrm{m}_{K}=$ 3.0 mag. If we assume for a cool star that the total luminosity, $\mathrm{L}$, is given by $\mathrm{L}=3 \nu \mathrm{L}_{\nu}$ for the emission near $2.2 \mu \mathrm{m}$ [the $\mathrm{K}$-band], and if we assume that $\mathrm{m}_{K}=0.0$ mag corresponds to $620 \mathrm{Jy}$ (Beckwith et al. 1976), then stars with $\mathrm{L}>10^{5} \mathrm{~L}_{\odot}$ have $\mathrm{M}_{K}<-10$. In the abseace of interstellar extinction, the TMSS was therefore complete for identifying luminous red supergiants to a distance of $4 \mathrm{kpc}$ from the Sun. With an average extinction of $0.15 \mathrm{~K}$ $\mathrm{mag} / \mathrm{kpc}$ (Jura, Joyce and Kleinmann 1989), we expect the TMSS to have been reasonably complete to a distance of about $3 \mathrm{kpc}$ for stars with $\mathbf{M}_{K}<-10$. Optical spectroscopy for essentially all the stars in the TMSS has been reported (see Bidelman 1980). We can therefore be reasonably confident that we can identify all the red supergiants in the solar neighborhood; at least those stars in the $75 \%$ of the sky covered by the TMSS.

The difficult task is to separate the stars with $\mathrm{L}>10^{5} \mathrm{~L}_{\odot}$ from those red giants with lower luminosities. The fluxes with appropriate corrections for extinction can be well measured; the major uncertainty in determining the luminosity is the distance. In order to include only the high luminosity stars, we restrict our analysis to stars that lie in clusters or otherwise have well-determined distances.

Jura and Kleinmann (1990b) have identified 21 mass-losing red supergiants (20 M-type, one $\mathrm{G}$ type, $\mathrm{L}>10^{5} \mathrm{~L}_{\odot}$ ) within $2.5 \mathrm{kpc}$ of the Sun. The $\mathrm{G}$ star is $\rho$ Cas; the M stars are all well known including such famous objects as VX Sgr, NML Cyg, $\mu$ Cep, $\alpha$ Ori and VY CMa. The distribution of these stars projected onto the plane of the Milky Way is shown in Figure 1. In marked contrast to the spatial distribution of the $45 \mathrm{~W}-\mathrm{R}$ stars within 2.5 $\mathrm{kpc}$ of the Sun (Conti et al. 1983, van der Hucht et al. 1988, there is no concentration of the red supergiants towards the Galactic Center region.

Because there are 21 stars that lie within a circle of radius $2.5 \mathrm{kpc}$ centered on the Sura, we find that projected onto the galactic plane the surface density of these red supergian $\hat{\imath}$ 
stars is about $1 \mathrm{kpc}^{-2}$. It seems most unlikely that we have missed more than half of all the relevant stars, and so an upper limit to the surface density of these stars in the solar neighborhood is $2 \mathrm{kpc}^{-2}$.

Miller and Scalo (1979) estimate that the birthrate of stars with $M>20 M_{\odot}$ in the solar neighborhood is about $510^{-6} \mathrm{kpc}^{-2} \mathrm{yr}^{-2}$. This implies that the $\mathrm{M}$ supergiants spend between about $210^{5}$ and $410^{5} \mathrm{yr}$ in this phase. According to Chiosi and Maeder (1986), stars with initial solar masses of 25 and $40 \mathrm{M}_{\odot}$ are predicted to have lifetimes in the Heburning phase with effective temperatures less than $6300 \mathrm{~K}$ of $4.610^{5}$ and $1.210^{5} \mathrm{yr}^{-1}$, respectively. Within the uncertainties, there is good agreement between the theoretical and derived lifetimes.

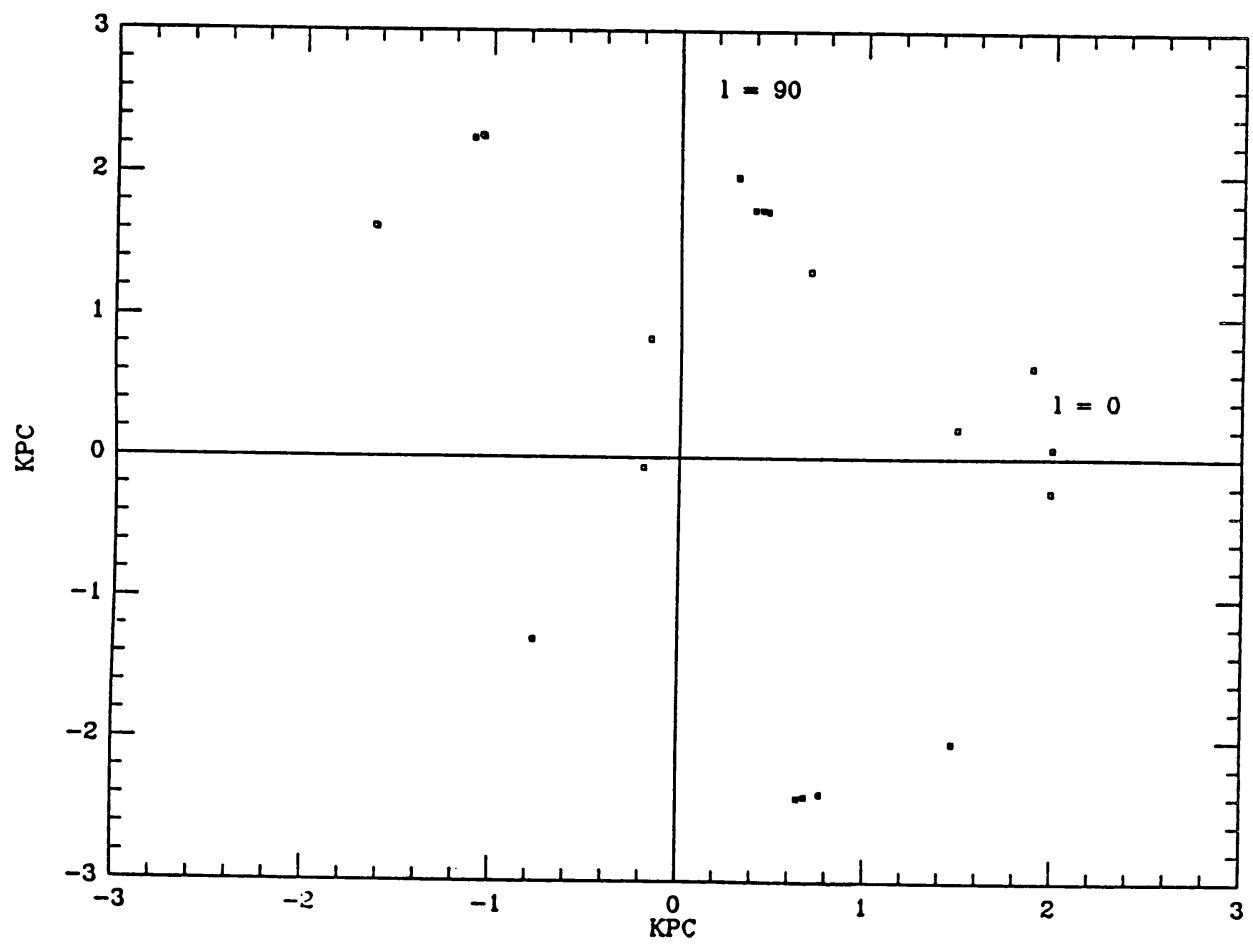

Fig. 1. Plot of the spatial distribution of the 21 red supergiants from Jura and Kleinmann (1990b) projected onto the plane of the Milky Way. The Sun is located at the center of the plot.

\section{MASS LOSS RATES}

The mass loss rates are inferred from the $60 \mu \mathrm{m}$ emission measured by IRAS and a standard model for the dust emissivity and dust to gas ratio. If the fraction of dust is very different from the assumed value - as may be the case for $\mu$ Cep (Le Borgne and Mauron 1989) 
and/or $\alpha$ Ori, (Jura and Morris 1981, Glassgold and Huggins 1986) then our inferred mass loss rates may not be correct.

For most stars, the amount of circumstellar dust can be determined relatively accurately from the amount of infrared emission. However, estimates of the amount of gas are more uncertain because it is usually only possible to measure directly the amount of trace species such as $\mathrm{CO}$ and then extrapolate to the amount of hydrogen. Consequently, our knowledge of the dust to gas ratios is uncertain. However, there are two stars where, because of special circumstances, the outflowing gas is photoionized, and it is possible to infer the amount of hydrogen that is being lost from its radio free-free emission. One such example is the very luminous star NML Cyg ( $L=510^{5} \mathrm{~L}_{\odot}$, Morris and Jura 1983), where it appears that the dust to gas ratio by mass is close to the value of 0.01 characteristic of the interstellar medium since the inferred mass loss rate derived from interpreting the infrared emission with this assumption is $1010^{-5} \mathrm{M}_{\odot} \mathrm{yr}^{-1}$ while modelling the radio free-free emission gives $610^{-5} \mathrm{M}_{\odot} \mathrm{yr}^{-1}$ (Jura and Kleinmann 1990b).

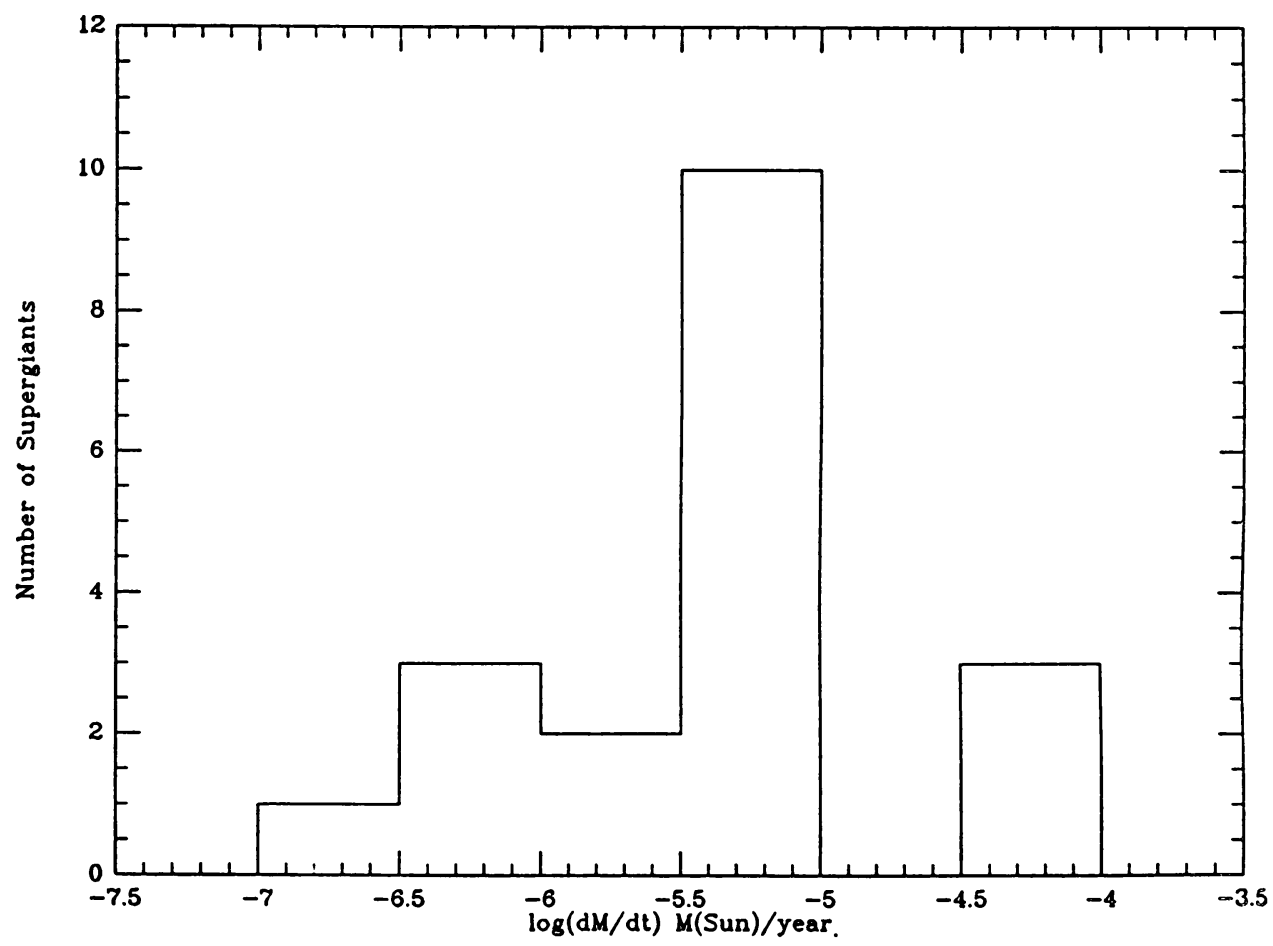

Fig. 2. Histogram of the mass-loss rates of the supergiants in Jura and Kleinmann (1990b).

The other example is the $M$ supergiant $\alpha$ Sco which is not quite luminous enough $\left(T_{2}=\right.$ $510^{4} \mathrm{~L}_{\odot}$, van der Hucht, Bernat and Kondo 1980) to be listed in our catalog. This star 
is losing mass, and because it has an early-type companion, much of the circumstellar gas is ionized. An accurate measurement of the gas loss rate is $210^{-6} \mathrm{M}_{\odot} \mathrm{yr}^{-1}$ (Hjellming and Newell 1983). Using the known distance, fluxes, luminosity and outflow velocity, and equation (2) of Jura and Kleinmann (1990b), the observed IRAS dust emission gives a total gas loss rate of $11^{-7} \mathrm{M}_{\odot} \mathrm{yr}^{-1}$ if we assume a "standard" dust to gas ratio of 0.01 by mass. [We know that the infrared emission from this star is produced by dust even though $\mathrm{F}_{\nu}$ varies as $\nu^{2}$ as from a photosphere because ground-based observations indicate silicate emission near $10 \mu \mathrm{m}$ from this star (Merrill and Stein 1976).] The way to reconcile the two discrepant results for the mass loss rate is to argue that the dust abundance around $\alpha$ Sco is lower than in the interstellar medium. In any case, with the assumption of a standard gas to dust ratio similar to that in the interstellar medium, a histogram of the mass loss rates for the different stars is shown in Figure 2.

The luminosities of the red supergiant stars vary by a factor of 5: between 1 and $510^{5}$ $\mathrm{L}_{\odot}$; in contrast, the range in the mass loss rates is much greater and amounts to a factor of 100. The two stars with the highest inferred mass loss rates are NML Cyg and VY CMa, both of which have values near $10^{-4} \mathrm{M}_{\odot} \mathrm{yr}^{-1}$, but a number of the supergiants have mass loss rates of about $10^{-6} \mathrm{yr}^{-1}$.

We do not fully understand the physics of the mass loss from the red supergiants. However, in all cases, it appears that radiation pressure on grains can be important since we find that

$$
v d M / d t<L / c
$$

The inferred mass loss rates indicate that mass loss is important in the evolution of these stars. If a star loses between $10^{-5}$ and $310^{-5} \mathrm{M}_{\odot} \mathrm{yr}^{-1}$ during the $\sim 310^{5} \mathrm{yr}$ that it might spend in this phase of its evolution, the total mass that is lost is in the range of 3 to $10 \mathrm{M}_{\odot}$. This mass that is lost is not negligible in the evolution of the star if it began with $20 \mathrm{M}_{\odot}$

The mass-return from the red supergiants is distinctly different from that of the W-R stars. The typical outflow speed from the red supergiants is perhaps $20-30 \mathrm{~km} \mathrm{~s}^{-1}$; a factor of 100 smaller than for the W-R stars. The red supergiants are all oxygen-rich while some of the W-R stars are carbon-rich. The grains around the red supergiants can be comparable in size to interstellar grains; perhaps $0.1 \mu \mathrm{m}$ in radius (see Herbig 1972, Jura 1975 for an analysis of the optical scattering properties of the grains around VY CMa). All the W-R stars which have grains are carbon-rich, and it is likely that these grains are quite small [< $0.01 \mu \mathrm{m}$ ] since they are driven supersonically through the wind by radiation pressure, and if they were larger, they would be destroyed by sputtering produced by collisions with the ambient gas (see, for example, Williams, van der Hucht and The' 1987).

The total mass-loss from all 21 stars identified by Jura and Kleinmann (1990b) is $310^{-4}$ $\mathrm{M}_{\odot} \mathrm{yr}^{-1}$. Over half of this total is from the two stars with the highest mass loss rates: NMI, Cyg and VY CMa. The total mass loss rate projected onto the galactic plane is somewhere between $11^{-5}$ and $310^{-5} \mathrm{M}_{\odot} \mathrm{kpc}^{-2} \mathrm{yr}^{-1}$. In contrast W-R stars return about $610^{-5} \mathrm{M}_{\odot}$ $\mathrm{kpc}^{-2} \mathrm{yr}^{-1}$. Therefore, the W-R stars return more mass to the interstellar medium than do the red supergiants.

As noted above, it seems that the W-R stars are much more concentrated towards the center of the Milky Way than are the red supergiants. Therefore, although the W-R stars dominate the average return of mass from massive stars to the interstellar medium in the solar neighborhood, outside the Solar Circle, the red supergiants may contribute as much 
matter into the interstellar medium as do the W-R stars.

This work has been partly supported by NASA. I thank Dr. Susan Kleinmann for many useful conversations.

\section{REFERENCES}

Abbott, D. C., and Conti, P. S. 1987, Ann. Rev. Astr. Ap., 25, 113.

Beckwith, S., Evans, N. J., Becklin, E. E., and Neugebauer, G. 1976, Ap. J., 208, 390.

Bidelman, W. P. 1980, Pub. Warner and Swasey Obs., 2, No. 6.

Chiosi, C., and Maeder, A. 1986, Ann. Rev. Astr. Ap., 24, 329.

Conti, P. S., Garmany, C. D., De Loore, C., and Vanbeveren, D. 1983, Ap. J., 274, 302.

Elias, J. H., Frogel, J. A., and Humphreys, R. M. 1985, Ap. J. Suppl., 57, 91.

Glassgold, A. E., and Huggins, P. J. 1986, Ap. J., 306, 605.

Herbig, G. H. 1972, Ap. J., 172, 375.

Hjellming, R. M., and Newell, R. T. 1983, Ap. J., 275, 704.

Humphreys, R. M. 1978, Ap. J. Suppl., 38, 309.

Humphreys, R. M., and McElroy, D. B. 1984, Ap. J., 284, 565.

Iben, I., and Renzini, A. 1983, Ann. Rev. Astr. Ap., 21, 271.

Jura, M. 1975, Astr. J., 80, 3.

Jura, M. 1989, in Evolution of Peculiar Red Giant Stars, IA U Colloquium 106, H. R. Johnson and B. Zuckerman, eds., (Cambridge: Cambridge University Press), p. 339.

Jura, M., Joyce, R. R., and Kleinmann, S. G. 1989, Ap. J., 336, 924.

Jura, M., and Kleinmann, S. G. 1990a, Ap. J., 351, 583.

Jura, M., and Kleinmann, S. G. 1990b, Ap. J. Suppl., in press.

Jura, M., and Morris, M. 1981, Ap. J., 251, 181.

Le Borgne, J. F., and Mauron, N. 1989, Astr. Ap., 210, 198.

Maeder, A. 1981, in The Most Massive Stars, S. D.'Odorico, D. Baade, and K. Kjar eds.

(Garching: ESO), p. 173.

Massey, P. 1981,Ap. J., 246, 153.

Merrill, K. M., and Stein, W. A. 1976, P.A.S.P., 88, 285.

Miller, G. E., and Scalo, J. M. 1979, Ap. J. Suppl., 41, 513.

Morris, M., and Jura, M. 1983, Ap. J., 267, 179.

Neugebauer, G., and Leighton, R. B. 1969, Two Micron Sky Survey (NASA SP-3047).

Olofsson, H. 1989, in Evolution of Peculiar Red Giant Stars, IAU Colloquium 106, H. R.

Johnson and B. Zuckerman, eds. (Cambridge: Cambridge University Press), p. 321.

Schulte-Ludbeck, R. E. 1989, Astr. J., 97, 1471.

van der Hucht, K. A., Bernat, A. P., and Kondo, Y. 1980, Astr. Ap., 82, 14.

van der Hucht, K. A., Hidayat, B., Adminranto, A. G., Supelli, K. K., and Doom, C. 1988, Astr. Ap., 199, 217.

Williams, P. M., van der Hucht, K. A., and The', P. S. 1987, Astr. Ap., 182, 91.

White, N. M., and Wing, R. F. 1978, Ap. J., 222, 209. 


\section{DISCUSSION}

Underhill: Can the picture be turned around? Say, WR stars are young objects in not fully dispersed molecular dust clouds. Sedlmayr has noted that having a disk helps dust to form. I would suggest that none of the observations discussed sofar this morning indicate that WR stars are the end-products of massive stars, and relevant to the density of interstellar material. [But Sedlmayer also noted that he needs high densities, i.e. high mass loss rates, and high carbon abundances. (Eds).]

Jura: Our results do not demonstrate that red supergiants are pre-WR stars.

Walborn: This is a good investigation of one channel of WR formation, but one must keep in mind that there are at least two others: stars with initial masses greater than $50 M_{\odot}$ which never become red supergiants, and mass transfer binaries. In addition, it is possible that steady-state red supergiant mass loss is not the dominant mechanism of core stripping: some objects exhibit LBV-like shell episodes, and the entire RSG envelope might be lost in a final, very short timescale event. Finally, lest some be confused by Underhill's remarks, the space distribution of WR stars is entirely inconsistent with a premain sequence interpretation: they are always found in evolved young regions, and never in regions of current star formation.

Humphreys: The three high mass loss red supergiants in your data set NML Cyg, VY CMa, and VX Sgr are all $\mathrm{OH} / \mathrm{IR}$ stars which are the high mass loss phase of the most evolved red supergiants. What was your $\dot{M}$ for IRC +10420 ? It is higher than the CO $\dot{M}$.

Jura: IRC +10420 was not included in our sample because it is too far away: the inferred mass loss rate is close to $10^{-3} M_{\odot} y r^{-1}$.

Sreenivasan: You considered only winds and mass loss from radiation pressure active on grains. Not all red supergiants become WR stars and not all stars become red supergiants. Formation of the WR phase is regulated by two time-scales, one for peeling the outer layers of the star and the other for hydrogen to burn to helium. Red supergiants have extensive outer convection zones and have non-thermal winds like the Sun - probably much stronger. All these have to be taken into account before deciding whether or not a star becomes a post-red supergiant WR star. There are also other forms of mass loss beside the two mentioned, e.g., pulsation driven mass loss, etc.

Maeder: The nuclear lifetimes for stars in the red supergiant stage depend on the initial masses. The $\dot{M}$-rates for red supergiants are also likely to depend on luminosities. It is the combination of both dependences which determines the mass interval for stars evolving through red supergiant and then WR stages. Thus, please also give us the $\dot{M}-L$ relations for red supergiants.

Jura: We have so few stars in our sample that it is difficult to establish firm results. Let me just make one point of clarification. The broad-band infrared colours around the star are very sensitive to the spatial distribution of grains. Because, what we are doing is, we are comparing the amount of cold dust to the amount of warm dust when you are looking at, say, $16 \mu$ vs. 12 or $25 \mu$, and in fact, generally we find very good agreement, and it is rather exceptional to find disagreement, with $R^{-2}$ distributions of dust grains and $R^{-1.5}$ and $R^{-2.5}$ would produce very different infrared colours than we witness. So, at least for most of the stars we look at, the mass loss rate in the zone where we can probe it, which is not all that big a volume, but it is probably a 1000 light years or so, has been fairly constant.

Leitherer: An important parameter for stellar evolution models is the dependance of mass loss rates of metallicity. In the case of hot stars, theory predicts a dependence of $\dot{M} \sim$ 
$Z^{0.5-1.0}$, which may (or may not) be supported by observations. (1) Is there a theoretical prediction for red supergiants? (2) What is the observational evidence?

Humphreys: In the LMC and SMC, the red supergiants have smaller $10 \mu$ silicate features in the sense they are weaker in the LMC than in galactic red supergiants of comparable luminosity and weaker in the SMC stars than in the LMC. This could be either a metallicity effect or a consequence of lower $\dot{M}$, most likely a combination of both.

Michael Jura

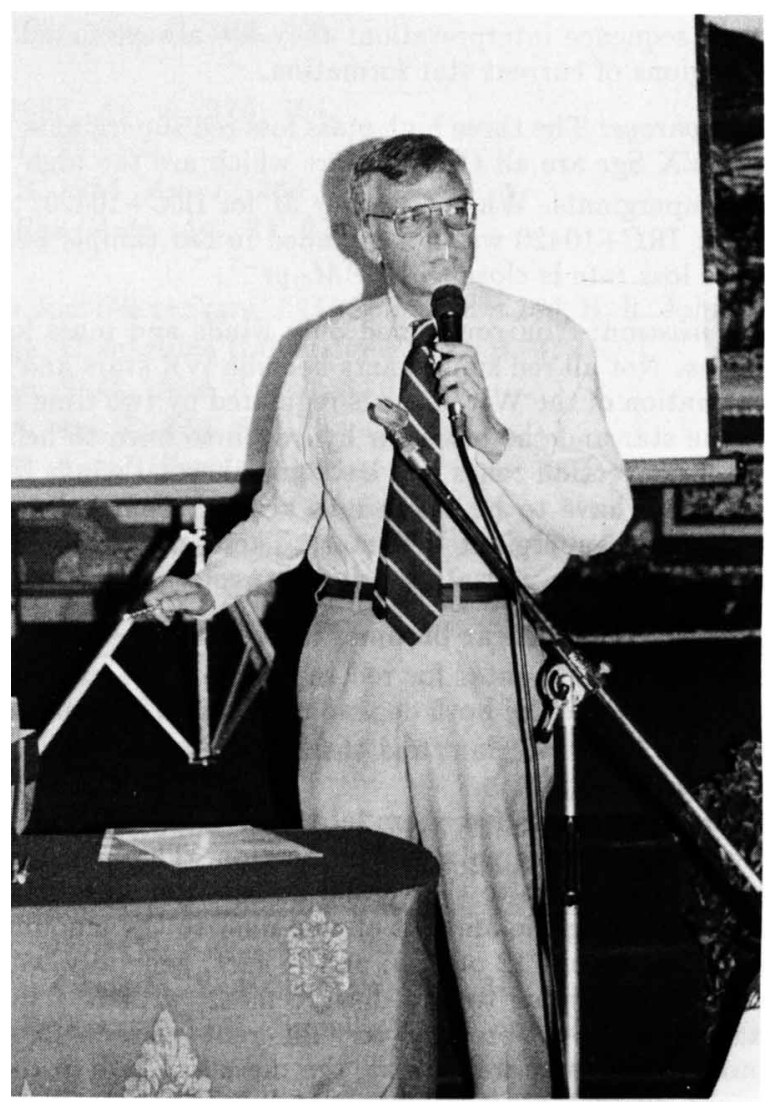

